\title{
Social support needs among patients with advanced breast cancer: sensitivity trumps substance
}

\author{
Sarah Kowitt, MPH, ${ }^{a}$ Shelly Yu, MPH, ${ }^{b}$ Anna Kate Owens, MSN, RN, FNP-BC, ${ }^{c}$ Lisa A \\ Carey, MD, ${ }^{\text {cd }}$ and Edwin B Fisher, PHD $^{a}$
}

aPeers for Progress and Department of Health Behavior, Gillings School of Global Public Health, University of North Carolina at Chapel Hill, North Carolina; ${ }^{b}$ The Advisory Board Company, Washington DC; 'Department of Medicine, University of North

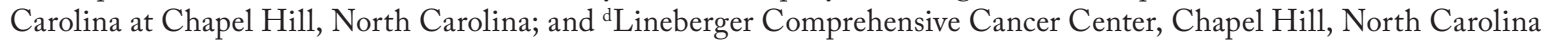

Background The importance of social support for cancer patients has been established in previous studies. However, much of the existing research has identified associations between general measures of social support and various health indicators. Nevertheless, some research has begun to suggest the utility of more nuanced understandings of how patients receive and use social support. Objective To examine the roles of nondirective (ie, support that accepts recipients' feelings and is cooperative with their plans) and directive support (ie, support that prescribes "correct" choices and feelings) as well as social support needs and desires among patients with advanced breast cancer.

Methods We conducted semi-structured interviews (qualitative method) with 8 patients with stage IV breast cancer to collect qualitative information about the disease-related challenges they faced, the support they received from their families and medical teams, and the appropriateness of directive and nondirective support. In addition, we used the 14-item Hospital Anxiety and Depression Scale (HADS) to assess clinically relevant cut-offs for anxiety and depression and the 16-item Social Support Inventory to assess the provision of nondirective and directive social support to the patients (quantitative method).

Results Qualitative findings suggested that there was considerable variability among patients' reports of social support provided by family, friends, and the medical team. From the qualitative data, patients reported directive support as more useful in times of acute need and emphasized the importance of supportive systems rather than supportive persons in providing emotional support. From the quantitative data, patients reported nondirective support as more typical of support received from both family and medical teams than directive support. On the HADS, 1 patient had a score of 9 on the anxiety subscale, above the score of 7 that is for mild anxiety. No patients scored above the criterion for mild depression, also a score of 7 .

Limitations Very small sample limits the ability to generalize findings.

Conclusions The right type of support for patients with advanced breast cancer is contingent on a range of variables, which suggests that the key characteristic of support may not be any particular feature, but the nuanced adjustment of its content and style of delivery to the patient's circumstances.

Funding Peers for Progress

$\mathrm{M}$ easures of social support and social connectedness have been linked to quality of life and overall health among those with cancer ${ }^{1,2}$ underscoring the importance of social support for cancer patients. ${ }^{3,4}$ Much research on social support in health and well-being has identified associations between summative or general measures of social support and various health indicators without inquiry into which features of social support are most important or the pathways through which their influence is expressed. However, some research, particularly in cancer, has begun to suggest the utility of more nuanced understandings of how social support is received and used by patients. For instance, using well-established distinctions among types of support, such as tangible, informational, or emotional, ${ }^{5}$ several studies have found that emotional support is most associated with better adjustment among cancer patients. ${ }^{6,7}$ In another study, emotional support about 2 months after diagnosis and both emotional and informational support about 7 months after diagnosis were associated with better quality of life among women with breast cancer. ${ }^{7}$

One way to characterize social support is by the type of support provided, leading to a distinction between:

Accepted for publication May 31, 2016. Correspondence: Sarah Kowitt, MPH; kowitt@email.unc.edu. Disclosures: The authors report no disclosures or conflicts of interest. JCSO 2016;14(12):509-514. (C2016 Frontline Medical Communications. doi: 10.12788/icso.0280. 
- Nondirective support that is accepting of recipients' feelings and choices, and cooperative with their plans.

- Directive support that prescribes "correct" choices and feelings (eg, You've got to look at the half of the glass that's full) and directs plans according to the provider's judgment of the best path to follow.

Research in both clinical and community samples has documented the benefits of nondirective support in terms of disease management, healthy lifestyles, and quality of life. .- $^{-}$ ${ }^{10}$ This has included studies of individuals with stages I and II lung cancer, ${ }^{9}$ and adults with multiple endocrine neoplasia. ${ }^{11}$ In general, nondirective support has been associated with better quality of life and disease management. ${ }^{8-10}$ However, a study that experimentally manipulated support types provided by an e-mail intervention for weight loss found that directive support led to greater weight loss than did nondirective support. ${ }^{13}$ Directive support also seems to be advantageous in circumstances of relatively acute or difficult challenges or in which a recipient is ill prepared to handle a challenge, which may include women after a hysterectomy or those with more advanced diabetes or systemic lupus erythematosus. ${ }^{13,14}$ Thus, it is possible that directive support may be beneficial for patients with advanced stage breast cancer.

Given the aforementioned findings, we aimed to examine the relationship between nondirective and directive support as well as social support needs and desires in patients with advanced (stage IV) breast cancer.

\section{Methods}

\section{Research design}

A concurrent mixed-methods design was used for this study, with quantitative measures administered to patients who participated in semi-structured interviews. ${ }^{15}$ We chose this design to allow for a more nuanced understanding of social support among breast cancer survivors. We collected data from 8 participants with advanced (stage IV) breast cancer recruited from the University of North Carolina Breast Cancer Center with the assistance of a nurse practitioner (AKO) who was assigned to approach for about participating in the study. The project was approved by the Institutional Review Board of the University of North Carolina at Chapel Hill, and informed consent was obtained from the participants before they enrolled in the study.

\section{Qualitative interview instrument}

In-depth semi-structured interviews were used to collect information on the challenges patients with breast cancer confront, the ways in which they were supported by their families and medical teams, and the appropriateness of directive and nondirective support. Topics were pre-determined by several of the authors (SY, EF, LC, AKO). Probes and open-ended questions were also used to ensure that participants' perspectives were fully explored. ${ }^{16}$ One member of the research team (SY) conducted all of the semistructured interviews with participants by telephone.

\section{Quantitative measures}

Hospital Anxiety and Depression Scale (HADS). The 14-item HADS was administered to the patients. The scale is used extensively with cancer patients because of its brevity and its ability to distinguish clinically relevant cut-offs for anxiety and depression. ${ }^{17}$ Items were scored on a scale of $0-3$, with 0 indicating least amount of anxiety and depression and 3 indicating the most. For both scales, scores of less than 7 indicate non-cases. ${ }^{18}$

Social Support Inventory (nondirective and directive support). A 16-item objective measure of nondirective and directive support was used to assess social support provision, with specific reference to support received from members of the patient's family and medical team (suppl 1). ${ }^{10}$ Each item was scored on a scale of 1-3, with 3 indicating that the listed type of support was very typical of support received, and 1 indicating the least typical. An example of a nondirective support item would be, How typical is it that [referent person/your medical team] asks you how you are doing? An example of a directive support item would be, How typical is it that [referent person/your medical team] pushes you to get going on things?

The Social Support Inventory has been used in a range research studies, including studies on diet and physical activity, ${ }^{19}$ multiple endocrine neoplasia, ${ }^{20}$ stage I and II lung cancer, ${ }^{9}$ condom use, ${ }^{21}$ and adaptive coping among patients with non-small-cell lung cancer. ${ }^{22}$ Findings from previous studies have demonstrated that this scale has high internal consistency with Cronbach's alpha of 0.89 and 0.74 for the nondirective and directive subscales, ${ }^{19}$ respectively, and consistency over time. ${ }^{12}$

\section{Analysis}

While data collection for the quantitative measures was ongoing, the qualitative data were analyzed to explore themes emerging in initial interviews. The interviews were audio-recorded, and the interviewer (SY) took field notes, which were corroborated with the recordings. Recordings and field notes were used as the basis for analytic memos and matrices, which organized findings by pre-identified deductive topics found in the interview guide and emergent inductive topics that had not been identified a priori. ${ }^{23}$ To ensure the reliability of findings and a nuanced understanding of results, emergent themes were discussed in team meetings.

Data analysis of the quantitative data included descriptive statistics to characterize levels of anxiety, depression, and nondirective and directive support from family and the medical team based on scores from the aforemen- 
tioned measures. After the quantitative and qualitative data analyses had been completed, the findings were integrated to yield a more thorough understanding of social support among participants. Given the small sample size, we emphasized the qualitative data, and the quantitative data were used to supplement the qualitative findings. We also did not analyze statistical relationships among measures because of the small sample size. Direct quotes from a participant are in quotation marks.

\section{Results}

\section{Participants}

A total of 8 women participated in this study. Six were white, 1 was African American, and 1 identified as Other. The average age for the patients was 61 years. All of them had stage IV breast cancer and had been diagnosed with cancer 3-32 years previously.

\section{Challenges associated with breast cancer}

The patients reported a number of challenges associated with advanced stage breast cancer. One consistent theme that emerged was the desire for normalcy. Stage IV breast cancer patients are rarely cured. Participants expressed frustration at this and indicated their desire to "be treated normally, just like everyone else," as one patient put it. Some of the patients had been living with their cancer diagnosis for years (though not necessarily with stage IV cancer for the entire time) and had psychologically moved on with their lives. One participant described her breast cancer as just "one small facet" of her life and "a backdrop to everything else." Participants also described psychological challenges in attempting to "stay upbeat" despite the physical challenge of not having energy, changes in appearance, and difficulties exercising. Other challenges they reported included having to deal with financial concerns, navigating the health care system, and creating or re-creating support systems. Results from the HADS indicated that 1 patient had a score of 9 on the HADS anxiety subscale, above the criterion of 7 for mild anxiety. No patients had a score above the criterion for mild depression, on the HADS depression subscale, also a score of 7 .

\section{Sources of support}

Patients reported support from a number of sources, including partners, family members, friends, and the medical team. For all patients, a variety of sources, rather than just one person or one type of support system, was important in providing support. Patients described how friends and peers were important in providing support, in addition to supportive family members and medical teams. For instance, a patient described how her husband was "always there for her," but sometimes she needed friends to talk to and they "weren't there 24 hours a day". Another patient described the help she received from other cancer patients.
As she stated,

... while nobody's cancer is the same, we all go through stages and they recognize each other's needs. We tell jokes and ask how each other is feeling. It's like two old friends having a conversation ... like preselected best friends.

In some instances, patients reported gaps in their support networks. For instance, one patient discussed how she lacked a supportive family network and how she was forced to "just deal with it [cancer] on my own." For her, a social worker was integral in connecting her with appropriate resources.

\section{Types of support}

Although there were obvious distinctions between the roles of the medical team and the supportive family or friends - for example, medical teams focused on providing treatment, whereas family members and friends provided assistance with activities of daily living and coping with the disease - there was also overlap in some of the support they offered. In particular, all of the patients spoke of the importance of emotional support from both family members and/or friends and the medical team. One patient spoke about friends who visited her to help her feel less scared and depressed when she was diagnosed with cancer. Another patient spoke about how important it was for friends and family to listen to her and "be there" to reassure her. In describing emotional support, patients often spoke of the value of implicit support ${ }^{25,26}$ that involved activities or the presence of others without discussion of one's problems. For instance, one patient spoke about the value that she derived from her friends and husband "just being there" and "taking me out to eat." At other times, patients reported needing explicit support, such as talking to friends and loved ones about their symptoms, concerns, or related issues.

\section{The appropriateness of nondirective and directive support}

Throughout the interviews, participants stressed the idea of independence and the importance of being able to "make up my own mind" and "not be a burden to others." When participants were asked their opinions of directive support (ie, directing plans according to the provider's judgment) they indicated that it was not appropriate because it prescribed "correct choices and feelings." They reported that they wanted to treat their illness on their own and receive support in their own way. In other words, they reported wanting a voice in deciding how they were treated. However, when participants were asked their opinion of specific examples of directive support (eg, providing clear advice on how to handle problems) many said the exam- 
ples were typical of the support they had received, especially from the medical team. Their responses suggest that directive support might be helpful in some situations, even though the general idea of directive support may seem less attractive to patients.

For example, one patient described not having a support system in place when she was diagnosed and reported having to "just deal with it [cancer]." She said it was helpful having the medical team, social worker, and nurse coordinator take charge, text her over weekends, keep her up to date on medications, make sure she could pay her bills, and so on. Another patient spoke about her son's suicide after her

TABLE Mean score (range, 1-3) ${ }^{a}$ on nondirective and directive support items from family and the medical team

\begin{tabular}{|c|c|c|}
\hline \multirow[b]{2}{*}{ Item } & \multicolumn{2}{|c|}{$\begin{array}{l}\text { Source of support } \\
\text { (mean score) }\end{array}$} \\
\hline & Family & $\begin{array}{l}\text { Medical } \\
\text { team }\end{array}$ \\
\hline \multicolumn{3}{|l|}{ Nondirective } \\
\hline $\begin{array}{l}\text { 1. Show interest in how you are } \\
\text { doing }\end{array}$ & 2.88 & 3.0 \\
\hline $\begin{array}{l}\text { 2. Make it easy for you to talk about } \\
\text { anything you think is important }\end{array}$ & 2.88 & 3.0 \\
\hline 3. Ask how you are doing & 3.0 & 2.88 \\
\hline 4. Are available to talk anytime & 2.88 & 2.88 \\
\hline 5. Ask if you need help & 2.88 & 2.75 \\
\hline $\begin{array}{l}\text { 6. Cooperate with you to get things } \\
\text { done }\end{array}$ & 3.0 & 3.0 \\
\hline $\begin{array}{l}\text { 7. Provide information so that you } \\
\text { understand why you are doing } \\
\text { things }\end{array}$ & 2.5 & 2.88 \\
\hline 8. Offer a range of suggestions & 2.63 & 3.0 \\
\hline Total mean score & 2.83 & 2.92 \\
\hline \multicolumn{3}{|l|}{ Directive } \\
\hline 1. Tell you to feel proud of yourself & 2.75 & 2.63 \\
\hline 2. Push you to get going on things & 1.88 & 2.25 \\
\hline $\begin{array}{l}\text { 3. Point out harmful or foolish ways } \\
\text { you view things }\end{array}$ & 1.5 & 2.25 \\
\hline $\begin{array}{l}\text { 4. Don't let you dwell on upsetting } \\
\text { thoughts }\end{array}$ & 2.5 & 2.13 \\
\hline 5. Solve problems for you & 2.13 & 2.38 \\
\hline 6. Take charge of your problems & 2.0 & 2.0 \\
\hline $\begin{array}{l}\text { 7. Give you clear advice on how to } \\
\text { handle problems }\end{array}$ & 2.5 & 3.0 \\
\hline 8. Tell you what to do & 1.25 & 2.13 \\
\hline Total mean score & 2.06 & 2.34 \\
\hline
\end{tabular}

${ }^{a} 1$, support is not at all typical; 2, support is somewhere in the middle; 3, support is very typical. diagnosis, and how useful it was for the clinical nurse to take charge of rescheduling appointments and for the oncologist to "ensure that everything was taken care of" in regard to her medications and meetings with counselors. Other patients spoke of the need for doctors and providers to "tell me what to do" when they were sick or needed help, such as changing an unhealthy diet or coping with treatment plans. Thus, directive support was described as useful when women faced acute challenges, such as an initial diagnosis or family emergency; uncertain circumstances; or challenges they did not know how to address. However, this was not to the exclusion of nondirective support (ie, accepting of the patients' feelings and choices and cooperative with their plans), which was beneficial in different circumstances. In speaking about value of nondirective support, women mentioned the importance of having others ask how they were doing and offer suggestions for different problems. For instance, when describing her medical team, one woman reported that they are there for her "emotionally and physically" and that they "listen to me and give me room to express myself."

These patterns of acceptance and need for, as well as reticence about directive support were reflected in the results of the 16-item Nondirective vs Directive Support Scale that was administered to the patients, in which nondirective support was reported to be more typical of the support received from family and medical teams than was directive support (Table). Of a possible score of 3 for each item (3, specific example of support is very typical; 2 , somewhat typical; 1 , not at all typical), the mean nondirective support scores for family and medical team were 2.83 and 2.92, respectively, whereas the mean directive support scores were 2.06 and 2.34, respectively. Overall, patients reported that medical teams provided slightly more nondirective and directive support than did family members. As noted, the range of scores for each item was 1 to 3 . Thus, participants reported considerable variability in these measures.

\section{Discussion}

The present study with 8 patients with advanced stage breast cancer found considerable variability among types of support and the importance of family, friends, others with breast cancer, and the medical team in providing that support. Although participants rated nondirective support as more typical of the support they received than directive support, there was considerable variability in that as well, suggesting that there was no "best" support but rather that its importance is attuned to the circumstances in which it is provided. Qualitative findings suggested patterns in how patients: reconciled confronting challenges with maintaining a "normal" life; decided what was and was not useful and appropriate with nondirective and directive support; emphasized the importance of emotional support; and spoke of the diversity of support from different sources. Implications for clinical practice and research are discussed below. 


\section{A paradox in support desired and support valued}

Although patients rated directive support as less typical of the support they had received and spoke of it as generally less useful, their responses to specific instances of support suggested that nondirective and directive support may each be useful at different times and in circumstances in the progression of cancer. This suggests that whether or not specific types of support are helpful may depend on the circumstance rather than the nature of support per se. These findings are consistent with previous studies that have demonstrated, for instance, that directive support may be more effective in helping patients lose weight in a 12-week program, ${ }^{12}$ but that nondirective support may be more helpful for ongoing metabolic control among those with diabetes. ${ }^{8}$

\section{Normalcy and independence: the impact of cancer on relationships}

Although the types of support needed throughout the different stages of cancer progression may vary, the patients in our study consistently spoke of a desire to maintain normalcy in their relationships with family and friends and remain independent. However, this was challenging as they reported that family and friends were simultaneously navigating new roles of taking them - the patients - to medical appointments, assisting with physical and mental stressors, and providing support as caregivers. Thus, while patients spoke of not wanting to "burden others" with their needs and of their desire to be treated as "normally" as possible, they all acknowledged that family, friends, and important others played key roles in their treatment and support, particularly in providing ongoing emotional support. The desire for independence was striking given participants' advanced stage of cancer and need for support from different sources. It is also noteworthy that participants were able to articulate a number of stressors and challenges without being symptomatic for depression or anxiety. This seems to parallel research in diabetes showing a disjunction between measures of depression and measures of distress related to the burdens of the disease and its management. ${ }^{26}$

Our findings also demonstrate that the experience of independence and the desire for it are not exclusive of the receipt of directive support at times. In other words, social support, including directive support can be the context for individual autonomy, not its contradiction. What seems to be important is whether assistance and structure of support matches the intensity of challenge and the recipients' skill for handling it. ${ }^{27}$ Thus, for patients with advanced breast cancer or other individuals dealing with complex medical conditions, directive support, which is more prescriptive, may even provide the context for individuals maximizing their independence and autonomy in areas in which their capacities remain substantial. Provided it is appropriately matched to patients' needs and their ability to receive it, caregivers and medical professionals may provide directive support without compromising independence.

\section{Social support sources: complementarity among multiple sources}

The tuning of support to the circumstances and challenges the patients faced was captured by one of the participants who said the "best support is having people know what type of support to give." However, without having experienced the illness, many supporters and caregivers have difficulty knowing what type of support to give. Perhaps family, friends, and clinicians should be sensitive to patients' needs for concrete, specific, directive, and/or tangible support especially when patients face new challenges (diagnosis, disease progression, substantial change in treatment) or challenges that exceed their skills (eg, managing new medications or navigating complexities of the health care system) but reserve nondirective, emotional, and motivational support for when patients are coping with ongoing challenges or conditions.

Understanding these issues may be helpful to family and friends or peer supporters. Peer support is support provided by patient peers or individuals with similar conditions or experiential knowledge of a condition. Some studies have found that peer support is particularly beneficial when patients are reluctant to ask for support out of concern for burdening others with problems or concerns and that it could be helpful for individuals with complex medical needs, such as advanced-stage cancer. ${ }^{25,28}$ In addition, all patients, to some extent, described how different sources of support were useful at different points along the disease continuum. Thus, rather than emphasizing the utility of one source of support over another, our findings demonstrated the complementarity among multiple, interacting sources of support.

\section{Limitations}

The major limitation of this study was the small sample size of 8 women with advanced breast cancer, which limits our ability to generalize the findings to patients from different populations, settings, or with different types of cancer. Also, because of the small number of participants, we chose not to use inferential statistics or estimate statistical significance of observed patterns. In addition, the interviews were conducted with patients with advanced stage breast cancer about their perceptions of received support. The addition of interviews with family members, friends, and clinical teams would have addressed the ecology of support surrounding the individual.

\section{Conclusions}

The key finding of this study was the variability of social support for patients with breast cancer - by provider or source, by circumstance, and by patient need. Specifically, partici- 
pants rated nondirective support as more typical but directive support was discussed as also being important in acute situations. In addition, participants spoke of the importance of emotional support and diverse sources of social support. Given the disease burden of cancer, especially for those with advanced-stage disease, these nuanced understandings of

\section{References}

1. Wilson KG, Chochinov HM, Skirko MG, et al. Depression and anxiety disorders in palliative cancer care. J Pain Symptom Manage Feb 2007;33(2):118-129.

2. Peters L, Sellick K. Quality of life of cancer patients receiving inpatient and home-based palliative care. J Adv Nurs Mar 2006;53(5):524-533.

3. Schroevers MJ, Ranchor AV, Sanderman R. The role of social support and self-esteem in the presence and course of depressive symptoms: a comparison of cancer patients and individuals from the general population. Soc Sci Med Jul 2003;57(2):375-385.

4. Stark D, Kiely M, Smith A, Velikova G, House A, Selby P. Anxiety disorders in cancer patients: their nature, associations, and relation to quality of life. J Clin Oncol Jul 15 2002;20(14):3137-3148.

5. Cohen S, Mermelstein R, Kamarck T, Hoberman HM. Measuring the functional components of social support. In: Sarason IG, Sarason BR, eds. Social support: theory, research, and applications. The Hague: Martinus Nijhoff; 1985:73-94.

6. Helgeson VS, Cohen S. Social support and adjustment to cancer: reconciling descriptive, correlational, and intervention research. Health Psychology 1996;15(2):135-148.

7. Arora NK, Finney Ruten LJ, Gustafson DH, Moser R, Hawkins RP. Perceived helpfulness and impact of social support provided by family, friends, and health care providers to women newly diagnosed with breast cancer. Psychooncology 2007;16:474-486.

8. Fisher EB, Jr., La Greca AM, Greco P, Arfken C, Schneiderman N. Directive and nondirective support in diabetes management. Int J Behav Med. 1997;4:131-144.

9. Walker MS, Zona DM, Fisher EB. Depressive symptoms after lung cancer surgery: their relation to coping style and social support. Psychooncology. 2006;15(8):684-693.

10. Harber KD, Schneider JK, Everard KM, Fisher EB. Directive support, nondirective support, and morale. J Soc Clin Psychol. 2005;24(5):691-722.

11. Kung AL, Moley JF, DeBeneditti MK, Walker MS, Fisher EB. Social support and stress among adults with multiple endocrine neoplasia type 2. Paper presented at the 2004 annual meeting of the Society of Behavioral Medicine, Baltimore, MD.

12. Gabriele JM, Carpenter BD, Tate DF, Fisher EB. Directive and nondirective e-coach support for weight loss in overweight adults. Ann Behav Med. 2011;41(2):252-263.

13. Fisher EB, Jr, Bickle C, Harber K, et al. Benefits of directive and nondirective support are moderated by severity of circumstances. Paper presented at the 1997 annual meeting of the Society of how social support emerges are important for delivering the optimal amount and type of care. More generally, the key characteristic of support may not be any particular feature of what it entails or how it is given, but the nuanced adjustment of its content and style of delivery to the circumstances of the individual receiving it: sensitivity trumps substance.

Behavioral Medicine, San Francisco, CA.

14. Jeffe DB, Freiman M, Fisher EB, Jr. Interactions of social support, stress, and type of menopause on depression. Paper presented at the 1996 American Psychological Association's Women's Health Conference, Washington, DC.

15. Leech NL, Onwuegbuzie AJ. A typology of mixed methods research designs. Quality \& Quantity. 2009;43(2):265-275.

16. Patton MQ. Qualitative evaluation and research methods. 2nd ed. Newbury Park, CA: Sage Publications Inc; 1990.

17. Zigmond AS, Snaith RP. The hospital anxiety and depression scale. Acta Psychiatr Scand. 1983;67(6):361-370.

18. Stern AF. The hospital anxiety and depression scale. Occup Med (Lond). 2014;64(5):393-394.

19. Stewart DW, Gabriele JM, Fisher EB. Directive support, nondirective support, and health behaviors in a community sample. J Behav Med. 2012;35(5):492-499.

20. Kung AL, Moley JF, DeBeneditti MK, Walker MS, Fisher EB. Social support and distress among adults with multiple endocrine neoplasia. Paper presented at the 2004 annual meeting of the Society of Behavioral Medicine, Baltimore, MD.

21. Gabriele JM, Williams C, Cavazos P, Fisher EB, Walker MS. Gender differences in relationships among social factors and self-efficacy for condom use. Annals of Behavioral Medicine. 2010;39:s141.

22. Walker MS, Zona DM, Fisher EB. Depressive symptoms after lung cancer surgery: their relation to coping style and social support. Psychooncology. 2006;15(8):684-693.

23. Fereday J, Muir-Cochrane E. Demonstrating rigor using thematic analysis: a hybrid approach of inductive and deductive coding and theme development. Int J Qual Methods. 2006;5(1): 80-92.

24. Kowitt SD, Urlaub D, Guzman-Corrales L, et al. Emotional support for diabetes management: an international cross-cultural study. Diabetes Educ. 2015;41(3): 291-300.

25. Kim HS, Sherman DK, Ko D, Taylor SE. Pursuit of comfort and pursuit of harmony: culture, relationships, and social support seeking. Pers Soc Psych Bull. 2006;32(12):1595-1607.

26. Snoek FJ, Bremmer MA, Hermanns N. Constructs of depression and distress in diabetes: time for an appraisal. Lancet Diabetes Endocrinol. 2015;3(6):450-460.

27. De Charms R. Personal causation: the internal affective determinations of behavior. St. Louis, Missouri. Academic Press; 1968.

28. Fisher EB, Ballesteros J, Bhushan N, et al. Key features of peer support in chronic disease prevention and management. Health Affairs. 2015;9: 1523-1530. 